\title{
Huéspedes definitivos de Spirometra mansonoides (Cestoda, Diphyllobothriidae) en el Perú
}

\author{
Definitive hosts of Spirometra mansonoides (Cestoda, Diphyllobothriidae) in \\ Peru
}

Manuel Tantaleán* y Carmen Michaud**

Presentado: 19/05/2005

Aceptado: 22/06/2005

\section{Resumen}

Se realizó un estudio parasitológico en el zoológico Parque de Las Leyendas, Lima, Perú en el año de 1993. Se recolectaron 49 muestras de heces de carnívoros pertenecientes a cinco familias: Canidae, Ursidae, Procyonidae, Mustelidae y Felidae, éstas fueron procesadas usando métodos rutinarios para la búsqueda de huevos de helmintos. En las heces de tres especies de la familia Felidae, Puma concolor (puma andino y puma de la selva), Panthera onca (otorongo) y Leopardus pardalis (tigrillo) se identificaron huevos de Spirometra mansonoides.

Palabras claves: Spirometra mansonoides, céstode, felino, huésped definitivo, Perú.

\section{Abstract}

A parasitology survey was conducted at the Parque de Las Leyendas Zoo in Lima, Peru at 1993. Forty nine stool samples of animals comprehended among five families of carnivores (Canidae, Ursidae, Procyonidae, Mustelidae, and Felidae) were collected and processed using routine methods for helminth ova detection. Three species of Felidae, Puma concolor, (puma andino and puma de la selva), Panthera onca (jaguar) and Leopardus pardalis (ocelot) had been infected with Spirometra mansonoides.

Keywords: Spirometra mansonoides, cestoda, feline, definitive host. Peru.

\section{Introducción}

En nuestro medio se tiene escaso conocimiento sobre las especies parasitarias que se encuentran habitualmente en la fauna silvestre. El presente estudio se realizó en un grupo de animales salvajes en cautiverio en el zoológico Parque Las Leyendas en Lima, Perú, con el fin de determinar la presencia de parásitos gastrointestinales y establecer un plan de tratamiento apropiado. Algunos de los parásitos que se encuentran en animales de vida silvestre pueden permanecer en sus huéspedes cuando se encuentran en cautiverio debido a la autoreinfección o la infección cuando las condiciones sanitarias, el alimento o la presencia de huéspedes intermediarios o vectores lo per-

(*) Unidad de Post Grado. Facultad de Ciencias Biológicas. Universidad Nacional Mayor de San Marcos. Lima, Perú.

E-mail: Manuel Tantaleán mtantaleanv@hotmail.com

(**) Facultad de Medicina Veterinaria. Universidad Nacional Mayor de San Marcos. Lima, Perú. miten; por ejemplo, los primates no humanos se infectan con oxyuroideos porque estos parásitos tienen ciclo de vida directo, los pinnípedos lo hacen con las larvas de ascáridos presentes en los peces marinos que ingieren como alimento (Soulsby, 1987). Los carnívoros son reservorios de protozoos, nemátodes, céstodes y trematodes, algunos de los cuales pueden infectar al hombre (por ejemplo: Toxoplasma gondii, Lagochilascaris minor, Spirometra mansonoides, Paragonimus spp.) (Soulsby, 1987). Sin embargo, poco se conoce sobre los huéspedes intermediarios o definitivos de estos parásitos en el Perú tanto en la fauna que proviene de su ambiente natural como en la que se encuentra en cautiverio; por este motivo, iniciamos este estudio en las especies de mamíferos carnívoros alojadas en el zoológico Parque Las Leyendas. 


\section{Material y métodos}

Se colectaron 49 muestras de heces de carnívoros. Las muestras fueron recogidas en las primeras horas de la mañana, tres veces para cada ambiente en días alternos, preservadas en formalina tamponada al $5 \%$ y procesadas aplicando métodos rutinarios para la búsqueda de huevos de helmintos (Ash \& Orihel, 1991). En algunos casos se tomaron muestras representativas y aleatorias de un grupo de animales pues estos no se encontraban alojados individualmente. Se prestó especial atención a las muestras de felinos, debido a que podían revelar la existencia de parásitos zoonóticos como Paragonimus y Lagochilascaris.
Los animales investigados y número de muestras se presentan en la Tabla1. El material se encuentra depositado en el Laboratorio de Parasitologia de Fauna Silvestre de la Facultad de Ciencias Biológicas de la Universidad Nacional Mayor de San Marcos, con el número de registro 70 .

\section{Resultados}

De las 18 especies de mamíferos carnívoros estudiados, en sólo tres de la familia Felidae se identificaron huevos de Spirometra mansonoides: Puma concolor, puma andino (dos positivas de tres muestras, 66,67 \%) y puma de la selva (una positiva de dos, $50 \%$ );

Tabla 1. Especies de carnivoros del zoológico Parque de Las Leyendas investigados en el presente trabajo, se indica el número de individuos, sexo y muestras de heces colectadas.

\section{Familia Canidae}

Lycalopex culpaeus (zorro colorado, atoj),

Lycalopex sechurae (zorro de sechura),

\section{Familia Ursidae}

Tremarctos ornatus (oso de anteojos),

\section{Familia Procyonidae}

Procyon cancrivorus (osito lavador),

Nasua nasua (coatí de cola anillada),

Potos flavus (chosna),

Bassaricyon alleni (olingo),

Familia Mustelidae

Mustela frenata (comadreja),

Eira barbara (tayra),

Galictis vittata (hurón grande),

\section{Familia Felidae}

Puma concolor (puma andino),

Puma concolor (puma de la selva),

Herpailurus yaguarondi (yaguarundi),

Leopardus pardalis (tigrillo),

Leopardus wiedii (margay),

Leopardus tigrinus (oncilla),

Panthera onca (otorongo),

Oncifelis colocolo (gato montés),

${ }^{*}$ snd $=$ sexo no determinado
2 hembras, 3 machos,

2 muestras.

10 hembras, 9 machos,

2 muestras.

3 hembras,

1 muestra.

1 hembras, 1 machos,

2 muestras.

21 hembras, 14 machos, 1 snd*, 7 muestras

12 hembras, 5 machos, 2snd*, 4 muestras

1 machos,

1 muestra.

1 machos,

1 muestra.

4 hembras, 3 machos,

1 muestra.

4 hembras 1 machos,

1 muestra.

5 hembras, 3 machos,

3 muestras.

1 hembras, 1 machos,

2 muestras.

1 machos, 1 snd*,

2 muestras.

9 hembras 5 machos,

8 muestras.

7 hembras 5 machos,

1 machos,

3 muestras.

1 muestra.

2 hembras, 4 machos,

5 muestras

3 machos, 
Panthera onca (1 de 5 muestras, 20\%) y Leopardus pardalis (1 de ocho muestras, 12,5 $\%)$. Se desconoce el tiempo que estuvieron los animales en cautiverio. Dos Panthera onca nacidos en cautiverio, resultaron negativos.

\section{Discusión}

Spirometra mansonoides es un céstode cuya larva plerocercoide (espargano) producen la esparganosis humana y animal. La infección en el hombre se adquiere por la ingestión del primer (copépodo del género Cyclops) o segundo huésped intermediario (anfibios o reptiles) infectados con procercoides o plerocercoides respectivamente (Mueller, 1974), o por el uso de la carne cruda infectada con el espargano a manera de emplasto en heridas expuestas o en los ojos. La larva migra al ponerse en contacto con la piel del huésped y en el caso del ojo puede producir exoftalmo, inflamación en los párpados y úlceras en la córnea (Kron et al., 1991; Raether \& Hänel, 2003). El espargano usualmente migra en el subcutis y se aloja en el tejido conectivo de los músculos, abdomen, piernas o debajo del peritoneo o pleura, pero también puede ser encontrado en otros lugares del cuerpo, causando reacciones inflamatorias severas y fibrosis en diferentes tejidos ( Raether \& Hänel, 2003).

En varios países latinoamericanos se han descrito casos de esparganosis humana de localización subcutánea y ocular, (Botero \& Gómez, 1958; León et al., 1972; Guderian et al., 1990; de Roodt et al., 1993). Asimismo existen dos reportes de esparganosis cerebral que es considerada poco frecuente (Boero et al., 1991; Landero et al., 1991) y uno de localización testicular (Sakamoto et al., 2003). Del mismo modo, se reconocieron larvas plerocercoides de Spirometra en 4 especies de ofidios de Uruguay, Leimadophis poecylogirus, Listrophis d'Orbigny, Phylodrias patagoniense y Bothrops neuwiedii y en el anuro Leptodactylus ocellatus (Dei-Cas et al., 1976).

En el Perú, se ha informado de 2 casos de infección humana (Tantaleán, 1994) y una en un mono Saguinus mystax (pichico barba blanca), procedentes del departamento de Loreto ( Michaud et al., 2003); pero aun no se han determinado las especies de animales que actúan como huéspedes intermediarios, tampoco la prevalencia y los mecanismos de infección de este céstode. En algunos lugares de la región andina del país existe la costumbre de ingerir anfibios de los géneros Batrachophrynus, Lynchophrys y Telmatobius como parte de la alimentación. También se engullen especímenes de Bufo vivos como actos de valor durante los ritos previos a determinados actos dancísticos («danza de tijeras») en algunos departamentos altoandinos como Huancavelica, lo que importaría un riesgo de infección si se comprueba que tales vertebrados actúan como segundo huésped intermediario. También se conoce el uso de carne de estos vertebrados como cataplasmas para curar heridas, principalmente en la selva.

En fauna salvaje norteamericana se ha descrito como huéspedes definitivos de $S$. mansonoides a Felis rufus (bobcats) (Watson et al., 1981; Heidt et al., 1988) y a Ursus americanus (oso negro) (Crum et al., 1978) y al Procyon lotor (mapache) que además puede albergar formas larvarias (Harkema, 1964). Además se tiene un reporte de un estudio realizado en las islas de las Antillas Holandesas, Curaçao, Bonaire y Aruba en donde como parte de la fauna parasitológica normal en gatos domésticos se identificó a S. mansonoides (Rep, 1975).

En relación a los huéspedes definitivos en Perú, sólo conocíamos de Atelocynus microtis (perro de monte) y de un zorro (probablemente Lycalopex spp. ) de la región selvática y Cajamarca respectivamente (Tantaleán y Guerrero, 1982-88) por lo que el presente trabajo nos permite señalar tres huéspedes naturales de la región amazónica. Este resultado tiene importancia porque, precisamente, los casos de esparganosis humana hasta hoy conocidos provienen de esta región. Spirometra 
erinacei, causante de la esparganosis en el viejo mundo ha sido descrito como fauna parasitológica habitual en Panthera leo (león africano) en Tanzania (Muller-Graf, 1995; Muller-Graf et al., 1999), a pesar de tratarse de una especie diferente lo consideramos relevante porque establece la presencia del género Spirometra en felinos salvajes.

Es muy poco probable de que los felinos que resultaron positivos a Spirometra en este reporte se hayan infectado en cautiverio debido a que el ciclo biológico del parásito requiere de la presencia de dos hospederos intermediarios, un crustáceo y un anfibio o reptil, los cuales no forman parte de la biota donde el zoológico se encuentra ubicado. Los felinos fueron alimentados con carne de res y tomaban agua potable. La autoinfección o re-infección no ocurre con esta especie de parásito.

\section{Tratamiento}

Con respecto al tratamiento se tiene un reporte de haber usado Ivermectina (vías subcutánea y oral) en gatos parasitados naturalmente con $S$. mansonoides sin resultados positivos (Blagburn et al.,1987). En un paciente con esparganosis proliferativa no se obtuvo ningún efecto con mebendazole ni praziquantel (Moulinier et al., 1982), pero sí en un perro también con esparganosis proliferativa (Beveridge et al., 1998). En el caso de larvas, el único tratamiento disponible consiste en la extirpación quirúrgica (Valerio et al., 2004). Miyazaki (1991) recomienda para $S$. erinacei adultos tratamiento igual a Diphyllobothrium latum, el cual consiste en la administración de bithionol, paromomycin y niclosamida.

S. mansonoides es un céstode de gran importancia, no sólo porque el plerocercoide puede infectar al hombre y animales, sino también porque sus metabolitos ejercen una acción beneficiosa en el huésped. El plerocercoide de $S$. mansonoides produce un factor que se asemeja en su actividad a la hormona de crecimiento humana $(\mathrm{HCH})$, ya que estimula el crecimiento en el huésped, se une a los receptores de la $\mathrm{HCH}$, da reacción cruzada contra anticuerpos anti-HCH y tiene actividad semejante a la insulina y es lactogénica (Salem \& Pares, 1989).

Se recomienda seguir muestreando carnívoros silvestres en cautiverio y/o en su habitat natural, para continuar identificando huéspedes definitivos de $S$. mansonoides en nuestro medio. Asimismo, educar a la población local con respecto al riesgo que implica las costumbres de ingerir y usar en medicina tradicional los huéspedes intermediarios de este parásito.

\section{Agradecimientos}

Los autores expresan su agradecimiento a los directivos del Patronato Parque Las Leyendas (PATPAL) por el apoyo brindado durante la realización del presente estudio, al Dr. Eduardo Núñez y a los técnicos Srs. Santiago Dimas, Sergio Montes y Francisco Tito, por la ayuda invalorable durante la recolección de muestras y a Dr. Alfonso Gozalo por las sugerencias.

\section{Literatura citada}

Ash, L. R. and T.C. Orihel. 1991. Parasites: A guide to laboratory procedures and identification. Am. Soc. Of Clin. Path. Chicago, 328 pp.

Beveridge, I., S. C. Friend, N. Jeganathan, and J. Charles. 1998. Proliferative sparganosis in Australian dogs. Aust. Vet. J. 76: 757-759

Blagburn, B. L., C. M. Hendrix, D. S. Lindsay, and J.L. Vaughan. 1987. Antihelmintic efficacy of ivermectin in naturally parasitized cats. Am. J. Vet. Res. 48: 670-672.

Boero, A.M., P. Garaguso, and J. Navarre. 1991. A case of cerebral sparganosis in South America. Arq. Neuropsiquiatr. 49: 111-113

Botero, D. and J. J. Gómez. 1958. The first case of sparganosis in Colombia. Amer. J. Trop. Med. \& Hyg. 7: 597-599.

Crum, J.M., V.F. Nettles, and W.R. Davidson. 1978. Studies on endoparasites of the black bear (Ursus americanus) in the southeastern United States. J. Wildl. Dis. 14: 178-186.

Dei-Cas, E., N. Rodríguez, C. Botto, J. y J. Osimani. 1976. Larvas plerocercoides de Spirometra (Dibothriocephalidae) en el hombre y en animales silvestres de Uruguay. Rev. Inst. Med. Trop. Sao Paulo 18: 165-172.

de Roodt, A.R., G. Suárez, A. Ruzic, E. Bellegarde, M. Braun, and C.M. Blanco. 1993. A case of human sparganosis en Argentina. Medicina (B. Aires). 53: 325-328. 
Guderian, R., J. Roldan, A. Guevara, and J. Chico. 1990. Human sparganosis in Ecuador: report of a case in the province of Esmeraldas. Rev. Soc. Bras. Med. Trop. 23: 115-116.

Harkema, R., and G.C. Miller. 1964. Helminth parasites of the raccoon, Procyon lotor, in the southeastern United States. J. Parasit. 50: 60-66.

Heidt, G.A., R.A. Rucker, M.L. Kennedy, and M.E. Baeyens. 1988. Hematology, intestinal parasites, and selected disease antibodies from a population of bobcats (Felis rufus) in central Arkansas. J. Wildlife Dis. 24: 180-183.

Kron, M. A., R. Guderian, A. Guevara, and A. Hidalgo. 1991. Abdominal sparganosis in Ecuador; A case report. Am. J. Trop. Med. Hyg. 44: 146150.

Landero, A., F. Hernández, M.A. Abasolo, D.A. Rechy, and D. Nuñez. 1991. Cerebral sparganosis caused by Spirometra mansonoides. J. Neurosurg. 75: 472-474.

León, L.A., R. Almeida, and J.F. Mueller. 1972. A case of ocular sparganosis in Ecuador. J. Parasit. 58: 184-185.

Michaud, C., M. Tantaleán, C. Ique, E. Montoya, and A. Gozalo. 2003. A survey for helminth parasites in feral New World non-human primate populations and its comparison with parasitological data from man in the region. $\mathrm{J}$ Med. Primatol. 32: 341-345.

Miyazaki, I. 1991. Helminthic zoonosis. Int. Med. Found. Japan. Tokyo.

Moulinier, R., E. Martínez, J. Torres, O. Noya, B.A. de Noya, and O. Reyes. 1982. Human proliferative sparganosis in Venezuela: report of a case. Am. J. Trop. Med. Hyg. 31: 358363.

Muller-Graf, C.D., M. E. Woolhouse, and C. Packer. 1999. Epidemiology of an intestinal parasite (Spirometra spp.) in two populations of African lions Panthera leo. Parasitology. 118: 407-415.
Mueller, J.F. 1974. The biology of Spirometra. J. Parasit. 60: 3-14.

Mueller, J.F. 1975. On the occurrence of Spirometra mansonoides in South America. J. Parasit. 61: 774-775.

Rep B. H. 1975. Intestinal helminthes in dogs and cats on the Antillian Islands, Aruba, Curaçao, and Bonaire. Trop. Geogr. Med. 27: 317-323.

Sakamoto, T., C. Gutiérrez, A. Rodríguez, and S. Sauto. 2003. Testicular sparganosis in a child from Uruguay. Acta Trop. 88: 83-86.

Soulsby, E.J.L. 1987. Parasitología y enfermedades parasitarias de los animales domésticos. $7^{\mathrm{a}}$. Edición. Nueva editorial Interamericana, S. A. de C. V. México D.F., México

Tantaleán, M. 1994. Nuevos helmintos de importancia médica en el Perú. Rev. Per. Med. Trop. UNMSM. 8: 87-91.

Tantaleán, M. y C. Guerrero. 1982-88. Presencia de Spirometra mansonoides en el Perú. Bol. Peruano Parasit. 4-10: 46.

Torres, P. and L. Figueroa. 1982. Infection by Spirometra mansoni (Cestoda, Pseudophyllidea) in the south of Chile. Bol. Chile. Parasit. 37: 72-73.

Valerio, I., B. Rodríguez, y M. Chinchilla. 2004. Primer hallazgo de Spirometra mansoni en Felis domesticus de Costa Rica. Parasitol. Latinoam. 59: 162-166.

Watson, T. G., V. F. Nettles, and W. R. Davidson. 1981. Endoparasites and selected infectious agents in bobcats (Felis rufus) from West Virginia and Georgia. J. Wildl. Dis. 17: 547-554. 